
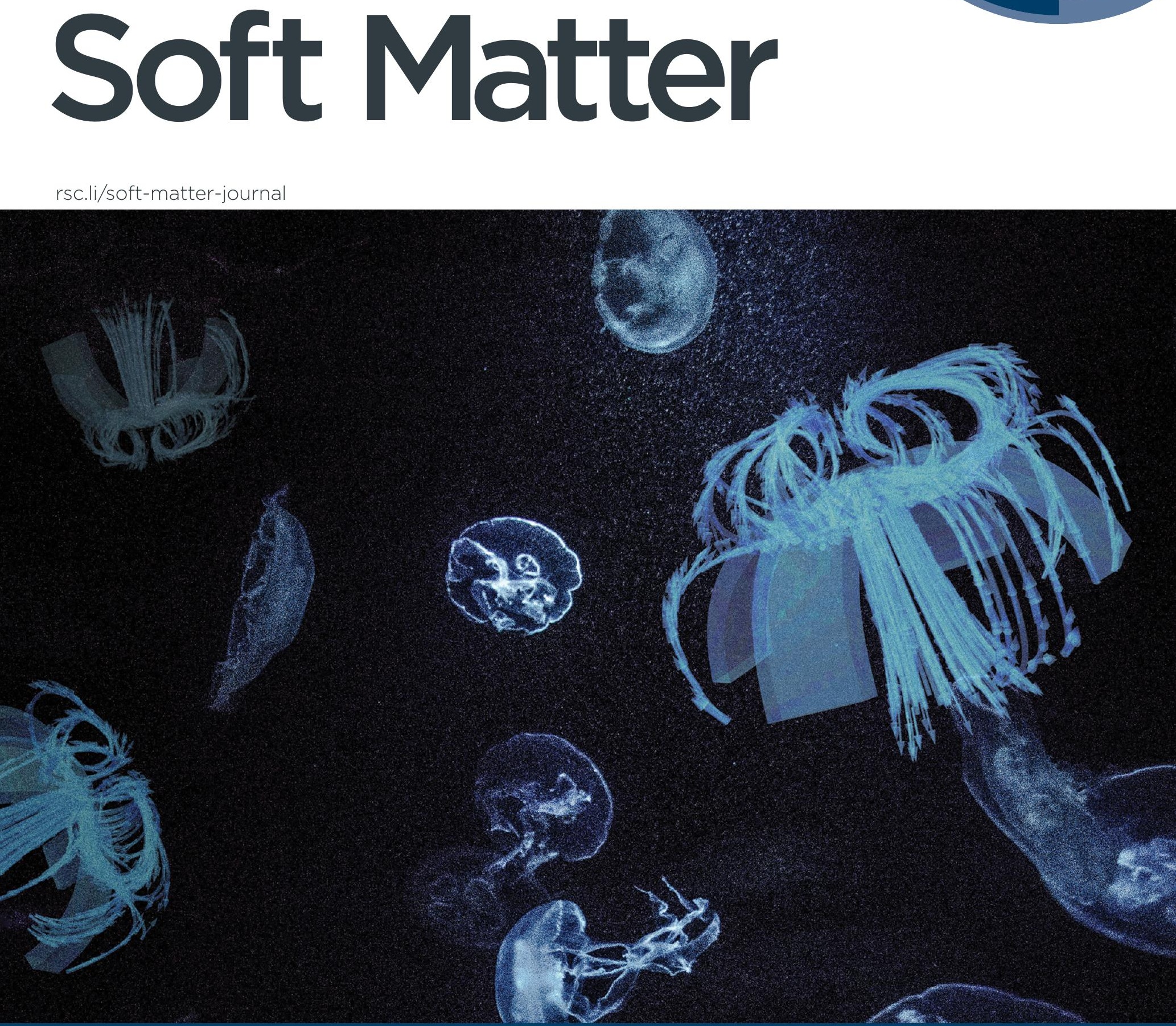

Themed issue: Soft Matter Emerging Investigators 2021

\title{
PAPER
}

Teng Zhang et al.

Magttice: a lattice model for hard-magnetic soft materials 


\title{
Soft Matter
}

Check for updates

Cite this: Soft Matter, 2021, 17, 3560

Received 15th September 2020 Accepted 29th November 2020

DOI: $10.1039 / \mathrm{d} 0 \mathrm{sm} 01662 \mathrm{~d}$

rsc.li/soft-matter-journal

\section{Magttice: a lattice model for hard-magnetic soft materials $\dagger$}

\author{
Huilin Ye, (D) ${ }^{a}$ Ying Li (D) ab and Teng Zhang (D) *cd
}

\begin{abstract}
Magnetic actuation has emerged as a powerful and versatile mechanism for diverse applications, ranging from soft robotics, biomedical devices to functional metamaterials. This highly interdisciplinary research calls for an easy to use and efficient modeling/simulation platform that can be leveraged by researchers with different backgrounds. Here we present a lattice model for hard-magnetic soft materials by partitioning the elastic deformation energy into lattice stretching and volumetric change, so-called 'magttice'. Magnetic actuation is realized through prescribed nodal forces in magttice. We further implement the model into the framework of a large-scale atomic/molecular massively parallel simulator (LAMMPS) for highly efficient parallel simulations. The magttice is first validated by examining the deformation of ferromagnetic beam structures, and then applied to various smart structures, such as origami plates and magnetic robots. After investigating the static deformation and dynamic motion of a soft robot, the swimming of the magnetic robot in water, like jellyfish's locomotion, is further studied by coupling the magttice and lattice Boltzmann method (LBM). These examples indicate that the proposed magttice model can enable more efficient mechanical modeling and simulation for the rational design of magnetically driven smart structures.
\end{abstract}

\section{Introduction}

Hard-magnetic soft materials, usually made by embedding hard magnetic neodymium-iron-boron ( $\mathrm{NdFeB}$ ) microparticles into soft matrix-like silicone elastomers, have attracted great attention due to their various remarkable features such as the response to remote external stimuli, fast actuation, excellent flexibility, and stretchability. ${ }^{1-7}$ Promising applications include soft robotics, ${ }^{8-14}$ machines and actuators, ${ }^{15-20}$ microfluidics, ${ }^{21-25}$ biomedical devices ${ }^{10,13,14,26-30}$ (e.g., endovascular neurosurgery ${ }^{10}$ and smart catheters ${ }^{29}$ ), and multifunctional architected materials and metastructures, ${ }^{8,25,31-37}$ just to name a few. The rapid developments of the field also call for efficient and accurate modeling and simulation platforms to rationalize the design, because these smart structures usually undergo very large and nonlinear deformation in complicated working environments, e.g., confined, enclosed spaces, combined liquid and solid terrain and brain's narrow

\footnotetext{
${ }^{a}$ Department of Mechanical Engineering, University of Connecticut, 191 Auditorium Road, Unit 3139, Storrs, Connecticut 06269, USA. E-mail: yingli@engr.uconn.edu; Fax: +1-860-4865088; Tel: +1-860-4867110

${ }^{b}$ Polymer Program, Institute of Materials Science, University of Connecticut, 97 North Eagleville Road, Unit 3136, Storrs, Connecticut 06269, USA ${ }^{c}$ Department of Mechanical and Aerospace Engineering, Syracuse University, Syracuse, NY 13244, USA. E-mail: tzhang48@syr.edu; Tel: +1-315-443-2969

${ }^{d}$ BioInspired Syracuse, Syracuse University, Syracuse, NY 13244, USA

$\dagger$ Electronic supplementary information (ESI) available: Simulation movies for turning and swimming of the magnetic robot. See DOI: 10.1039/d0sm01662d
}

and winding vasculature. ${ }^{9,14,20,38}$ For example, $\mathrm{Hu}$ et $a .^{9}$ made untethered small-scale robots that can perform various complicated forms of locomotion, such as walking, jumping, rolling, and swimming by designing a harmonic magnetization profile in a magneto-elastic, rectangular sheet. These locomotion modes require a sophisticated control scheme to maneuver the robot as highly nonlinear deformation and multiphysics couplings are inevitable. An efficient and accurate modeling platform can provide a solid foundation for identifying the optimum control scheme and reduce the experimental trial-and-error.

Among various numerical techniques, ${ }^{32-39}$ finite element methods (FEMs) are widely used to simulate the nonlinear and active deformation of ferromagnetic materials. ${ }^{32,39}$ For example, Zhao et $a .^{32}$ derived a finite element simulation scheme for the hardmagnetic soft materials and implemented it into the FEM software ABAQUS through a user-defined element (UEL). This robust and powerful platform has greatly promoted the computational contributions to the design of magnetic smart structures. However, it is still difficult to include the multiphysics interactions into the framework of UEL in ABAQUS, such as fluid-structure interactions. Besides, the commercial software's parallel simulation capabilities are also constrained by the setup of the computational environments and the availability of the research licenses. Lattice models are another kind of numerical techniques for simulating elastic solids that are efficient and easy to integrate with other methods for multiphysics problems. ${ }^{40-48}$ For example, lattice spring gel models have been applied to study the fluid-driven 
motion of microcapsules on compliant surfaces. ${ }^{44,49}$ Recently, the authors derived irregular lattice models directly from the FEM framework, ${ }^{46}$ and further coupled the lattice model with the lattice Boltzmann method (LBM) for complicated fluid-structure interactions (e.g., the platform of OpenFSI ${ }^{50}$ ). Although the simplicity and powerfulness of the lattice model make it very promising, there is still a lack of a rigorous derivation to include the magnetic forces.

In this work, we present a modeling and simulation platform of hard-magnetic soft materials that combines the advantages of the recently developed FEM schemes and lattice models, named 'magttice'. We show that the magnetic actuation can be incorporated into the existing lattice model as nodal forces, which can be pre-computed if the external magnetic field is uniform. We implement the magttice model into the open-source molecular dynamics package, LAMMPS, and leverage its highly parallelized simulation capability and the versatile simulation techniques for multiphysics problems, such as fluid-structure interactions, by coupling the magttice model with the LBM. We then apply the magttice model to investigate the folded deformation of an origami plate, nonlinear deformation, and swimming of small-scale robots made by using a magnetic strip. These examples indicate that the proposed magttice model can enable more efficient mechanical modeling and simulation for the rational design of magnetically driven smart structures.

\section{Computational model and benchmark}

For hard-magnetic soft materials under moderate deformation (i.e., strain less than $30 \%$ ), Zhao et al. ${ }^{32}$ have shown that the strain energy density $(U)$ can be expressed as the summation of elastic energy (i.e., the neo-Hookean model) and magnetic energy

$$
U=\frac{G}{2}\left(I_{1}-3\right)+\frac{K}{2}(\ln J)^{2}-G \ln J-\frac{1}{\mu_{0}} \mathbf{F}^{r} \cdot \mathbf{B}^{\text {applied }}
$$

where $G$ is the shear modulus, $K$ is the bulk modulus, $I_{1}=\lambda_{1}{ }^{2}+\lambda_{2}{ }^{2}+$ $\lambda_{3}{ }^{2}$ for plane strain deformation is the first invariant of the right Cauchy-Green deformation tensor, $\lambda_{i}, i=1,2,3$ is the principal stretches and $J$ is the determinant of the deformation gradient tensor $\mathbf{F}, \mu_{0}$ is the vacuum permeability, $\tilde{\mathbf{B}}^{r}$ and $\mathbf{B}^{\text {applied }}$ denote the residual and applied magnetic flux densities, respectively. Note that $\tilde{\mathbf{B}}^{r}$ is defined in the reference configuration. The strain energy density in eqn (1) is derived for ideal hard-magnetic soft materials, ${ }^{32}$ whose magnetic flux density is linearly related to the applied magnetic field. For more general cases, higher-order terms of the magnetic flux density should be included in the strain energy density function. ${ }^{39,51,52}$ For large deformation, the soft matrix will exhibit strain hardening (i.e., $30 \%$ for $\mathrm{PDMS}^{53}$ ) and thus requires modifying the elastic strain energy density in eqn (1). Since many applications utilize large geometry nonlinear deformation with small stretching or compression, we focus on the neo-Hookean model. It should also be noted that viscoelasticity is not included in the current model and has been shown to play an important role in the deformation of magnetic soft materials. ${ }^{54,55}$ All these nonlinearities in mechanical deformation and magnetic interactions are important and interesting research topics, which will be studied in our future work.

The lattice model is derived based on energy equivalence through comparing with the finite element model. In the framework of the FEM, the deformation gradient tensor $\mathbf{F}$ in an irregular element can be calculated as: ${ }^{56}$

$$
F_{i j}=\frac{\partial x_{i}}{\partial X_{j}}=x_{i}^{a} \frac{\partial N^{a}}{\partial X_{j}},
$$

where $N^{a}\left(X_{1}, X_{2}, X_{3}\right)$ is the shape function for a hexahedron element, $a=1,2, \ldots, 8$, and $i, j, k=1,2,3$. The energy associated with $I_{1}$ can be written as:

$$
V_{0} U_{I_{1}}=\int \frac{1}{2} \mu^{s}\left(x_{i}^{a} x_{i}^{b} \frac{\partial N^{a}}{\partial X_{j}} \frac{\partial N^{b}}{\partial X_{j}}-3\right) \mathrm{d} V_{0} .
$$

By rearranging the summations in eqn (3), the energy can be obtained in the lattice model (28 lattice springs in a general case Fig. 1(a)) with the form ${ }^{46}$

$$
U_{I_{1}}=\frac{G}{2}\left(I_{1}-3\right)=\frac{1}{2} V_{0}^{-1} \sum_{b=2, b>a}^{8} \sum_{a=1}^{7} k_{a b} r_{a b}{ }^{2}-G,
$$

where $\mathbf{r}_{a b}=\mathbf{x}^{a}-\mathbf{x}^{b}$. Comparing above two energy expressions, we can easily calculate $k_{a b}=-\int G \frac{\partial N^{a}}{\partial X_{j}} \frac{\partial N^{b}}{\partial X_{j}} \mathrm{~d} V_{0}, j=1,2,3$, $a=1,2, \ldots, 7, b=2,3, \ldots, 8$. The volumetric energy is calculated with an averaged volumetric strain (Fig. 1),

$$
U_{\mathrm{J}}=\frac{1}{2} K\left(\ln \left(V / V_{0}\right)\right)^{2}-G \ln \left(V / V_{0}\right),
$$

where $V$ and $V_{0}$ represent the lattice's volumes at the deformed and undeformed (reference) configurations, respectively. The spring constants $k_{a b}$ can be computed following the same procedure of calculating thestiffness matrix in the FEM. In short, the lattice model leverages FEM techniques to rewrite the strain energy inside a hexahedron element as a summation of energies associated with lattice stretching and volumetric changes. Note that the bulk modulus $K$ is usually not directly reported in the literature, and here we just set as $K=20 G$ to mimic the nearly incompressible condition of polymers and soft materials. ${ }^{57,58}$

For the magnetic energy density, it can also be precomputed as

$$
U_{\text {magnetic }}=V_{0}^{-1}\left(-f_{\mathrm{m}}\right)_{i}^{a} x_{i}^{a},
$$

where $\left(f_{\mathrm{m}}\right)_{i}^{a}=\frac{1}{\mu_{0}} \tilde{B}_{j}^{r} B_{i}^{\text {applied }} \int \frac{\partial N^{a}}{\partial X_{j}} \mathrm{~d} V_{0}, i, j=1,2,3, a=1,2, \ldots, 8$ can be considered as general nodal forces associated with the magnetic actuation (Fig. 1(a)). It is noted that eqn (6) is valid only for spatially uniform external magnetic field because additional forces should be considered due to the gradient of the field. ${ }^{59}$ For a given external magnetic field that is characterized by the applied magnetic flux density $\mathbf{B}^{\text {applied }}$, it is interesting to note that $\mathbf{f}_{\mathrm{m}}$ is a dead force under large deformation whose direction does not change along with the deformation. Therefore, the magnetic forces can be easily incorporated into 
(a)

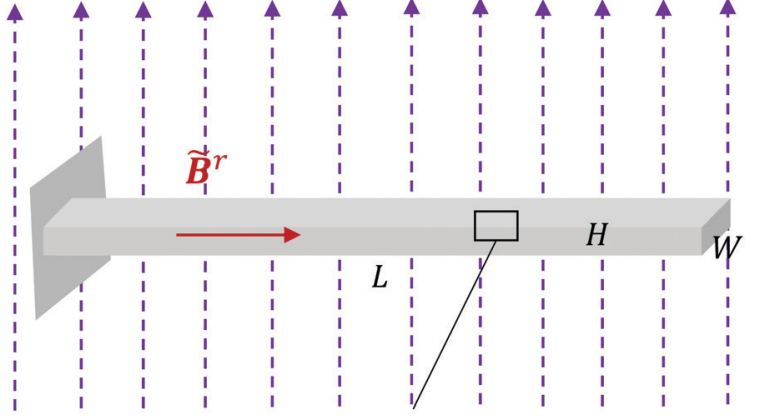

Magnetic lattice

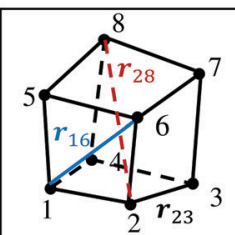

Stretching

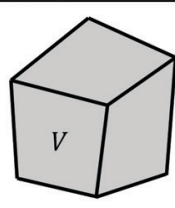

Volumetric

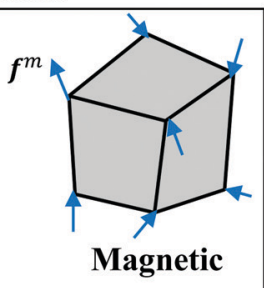

(b)

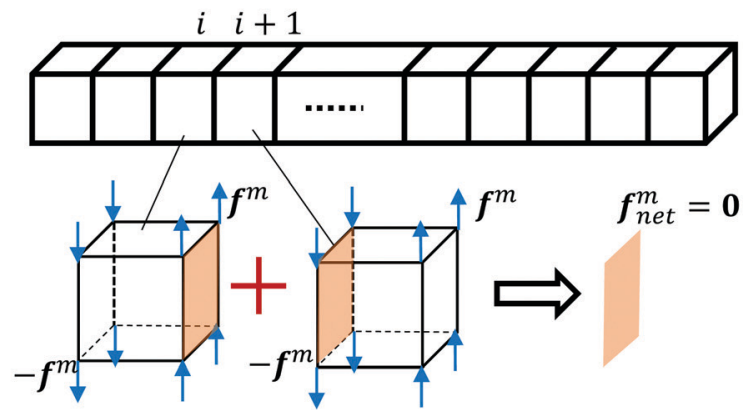

(c)

(d)
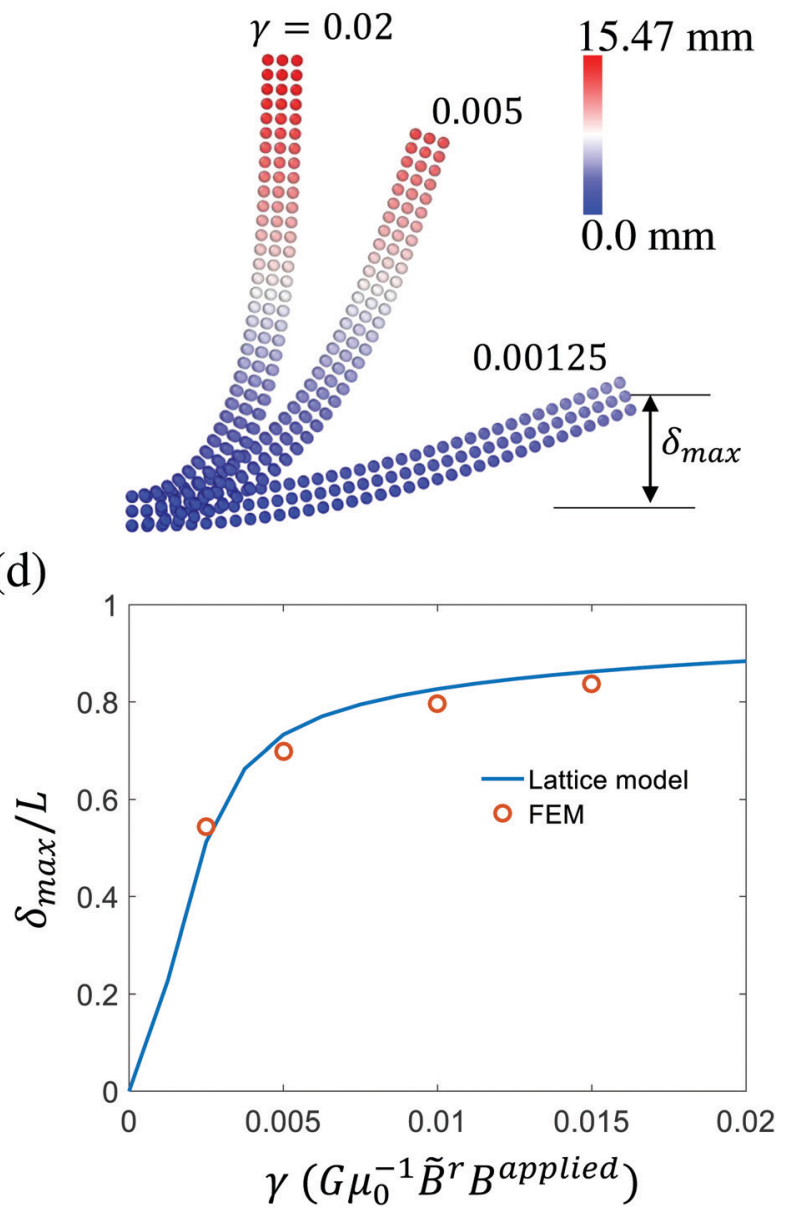

Fig. 1 Computational model and benchmark of the proposed magttice model. (a) Illustration of the proposed magttice model for ferromagnetic materials. (b) Diminished magnetic forces for the interior part of a beam with uniformly residual and external magnetic fields. (c) Deformed configurations of a representative beam (length $L=17.5 \mathrm{~mm}$, width $W=5 \mathrm{~mm}$, and height $H=1 \mathrm{~mm}$ ) at different external magnetic fields. The dimensionless variable $\gamma=G \frac{1}{\mu_{0}}\left|\widetilde{\mathbf{B}}^{r}\right|\left|\mathbf{B}^{\text {applied }}\right|$ characterize the effective strength of magnetic forces, in comparison to the elasticity. (d) The normalized maximum displacement as a function of $\gamma$. The finite element solution is taken from previous work done by Zhao et al. ${ }^{32}$

the same framework through added nodal forces in the job script of LAMMPS simulations. This enables us to directly simulate active deformations due to magnetic forces in OpenFSI ${ }^{50}$ which is based on LAMMPS and already has lattice models for pure mechanics structures. For temporally changing and spatially uniform external magnetic field, we will show in the following sections (Section 3) that the updates of the magnetic forces can be directly done within the LAMMPS script without re-running the integration in eqn (6). This will significantly simplify the simulations of the dynamic motions of magnetic robots, such as swimming in water.

Viewing the magnetic actuation as forces can also help simplify the modeling systems. Taking a ferromagnetic beam structure as an example with $\tilde{\mathbf{B}}^{r}=[1,0,0]^{\mathrm{T}}$ and $\mathbf{B}^{\text {applied }}=[0,0,1]^{\mathrm{T}}$ (Fig. 1(a) and (b)), the magnetic forces applied to the interior nodes will be cancelled out by summing the forces of the adjacent elements. This will lead to zero internal forces and non-zero nodal forces at the two ends, which is consistent with the recent observations in the modeling of the magnetic actuation of ferromagnetic beams with the elastic model. ${ }^{60} \mathrm{We}$ further simulate the deformed configurations of the representative ferromagnetic beam (length $L=17.5 \mathrm{~mm}$, width $W=$ $5 \mathrm{~mm}$, and height $H=1 \mathrm{~mm}$ ) using the proposed magttice model (Fig. 1(c)), where we have $G=330 \mathrm{kPa}, K=20 \mathrm{G}$ and $\frac{1}{\mu_{0}}\left|\widetilde{\mathbf{B}}^{r}\right|\left|\mathbf{B}^{\text {applied }}\right|$ as a tunable parameter. As shown in Fig. 1(d), our simulation results are in excellent agreement with FEM results reported by Zhao et al. ${ }^{32}$

\section{Applications to smart structures}

Since the magttice model has been validated through a benchmark of beam bending simulation, we further demonstrate its 
capability of handling nonlinear and coupling deformation of smart structures with inhomogeneous residual magnetic fields. These examples include origami plates and magnetic robots, which represent structures with discretely and continuously nonuniform residual magnetic flux density, respectively.

\subsection{Origami plate}

Miura-ori folds have been created by encoding alternating oblique patterns of ferromagnetic domains in a simple rectangular structure (Fig. 2(a)). This is a representative example of non-uniform spatial residual magnetic flux density. We adopt the same geometries (Fig. 2(a)) and material properties in a previous work, ${ }^{32}$ where we have $G=330 \mathrm{kPa}, \quad K=20 \mathrm{G}, \quad \frac{1}{\mu_{0}}\left|\widetilde{\mathbf{B}}^{r}\right|=81 \mathrm{kA} \mathrm{m}^{-1}$ and $\left|\mathbf{B}^{\text {applied }}\right|=200 \mathrm{mT}$. After discretizing the origami plate into a 3D lattice, we compute the magnetic forces at each node. We then let the structure deform under magnetic forces and relax to its final equilibrium configuration by adding damping in the simulation. As depicted in Fig. 2(b), the magttice model can successfully capture the Miura-ori folds. It is interesting to note that forces concentrate on the domain boundaries with zero values in the interior of the domains (Fig. 2(c)). This can provide guidance for developing more efficient reduced-order models, such as plates and shells with active components, to understand the ferromagnetic origami plates' mechanical behaviors with a much larger number of unit cells.

\subsection{Statically deformed configuration of the magnetic strip}

We next apply the magttice model to small-scale soft-bodied robots made by using magnetic strips with continuous and nonuniform residual magnetic flux density. We take the magneto-elastic, rectangular sheet in the work of $\mathrm{Hu}$ et $a l^{9}$ as a representative structure and first focus on the statically deformed configurations. As shown in Fig. 3 (the central schematic), a flat sheet is placed on a solid platform and has a harmonic magnetization profile with $\beta_{\mathrm{R}}=45^{\circ}$. We model the same robot used in their work, with length $L=3.7 \mathrm{~mm}$, width $w=1.5 \mathrm{~mm}$, and thickness $h=185 \mu \mathrm{m}$. The material properties are also obtained from the same work, such as $G=33 \mathrm{kPa}, K=20 \mathrm{G}$, density $\rho=1.86 \mathrm{~g} \mathrm{~cm}^{-3}$ and $\frac{1}{\mu_{0}}\left|\widetilde{\mathbf{B}}^{r}\right|=62 \mathrm{kA} \mathrm{m}^{-1}$. In the following discussions, we keep all

(a)

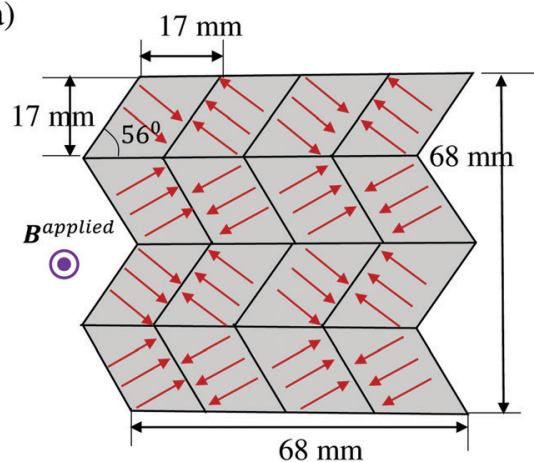

(b)

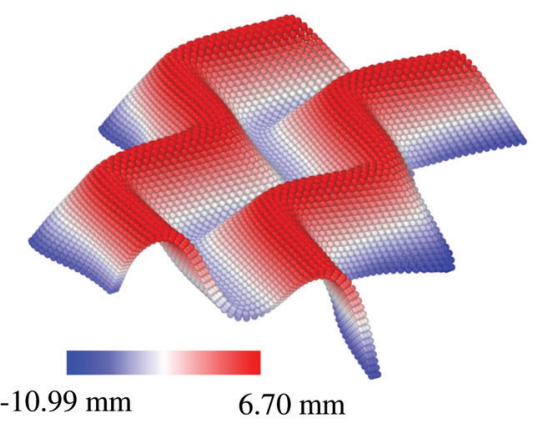

(c)

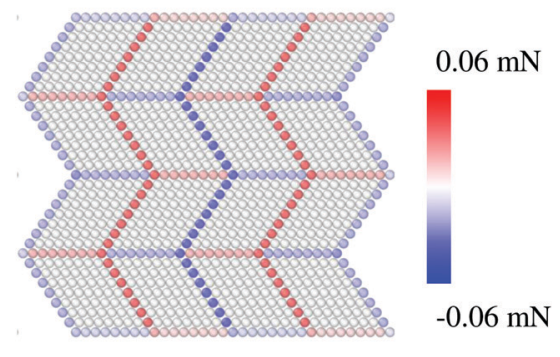

Fig. 2 Origami plate for Miura-ori folds. (a) The initial planar structure with an alternating ferromagnetic domain. The thickness of the plate is taken as $1 \mathrm{~mm}$. (b) The deformed Miura-ori folds. (c) The distribution of the magnetic forces. 
noticed for $\theta=90^{\circ}, 180^{\circ}, 270^{\circ}$, and $315^{\circ}$. These findings are all in excellent agreement with the analytical solutions and experiments in the previous study. ${ }^{9}$ However, the configurations for $\theta=135^{\circ}$ and $\theta=315^{\circ}$ are more complicated, which is neither a " $\mathrm{V}$ " nor a " $\mathrm{C}$ " shape. In addition, there is no rigid body rotation and two contact points between the robot and the platform.

\subsection{Rigid body motion of the magnetic strip}

The case with $\theta=135^{\circ}$ or $\theta=315^{\circ}$ seems an outlier in terms of the rigid body rotation and the deformation mode. It motivates us to further examine the effects of magnetic and gravity forces applied on the robot. We notice that there is a competition between the magnetic force and gravity. Focusing on the moment at point $A$, the magnetic force tends to generate rigid rotation to push the strip away from the ground, while the gravity creates moments to pull the strip down to the ground. Since the rigid body rotation is also coupled with the large nonlinear elastic deformation (Fig. 4(a)), we do not pursue the analytical formula of the moment applied to point A (center of the edge $(x=0, y=0)$ ) at a given deformed configuration. Instead, we quantify the competition between magnetic and gravitational forces (along the negative $y$ direction) by gradually tuning the value of density and the magnitude of the applied magnetic flux density $\left|\mathbf{B}^{\text {applied }}\right|$. When the rigid body rotation happens, only one contact point exists between the magnetic strip and platform, the tangential line of the valley of the "V" shape can be used as an indicator of the extent of the rigid body rotation. For the normal value of gravity on earth, no rigid body rotation is observed, even for a large magnetic magnitude as $20 \mathrm{mT}$ (Fig. 4(b) and (c)). When reducing the gravity by a factor of 2 , rigid body rotation begins when the magnetic magnitude is larger than $6.25 \mathrm{mT}$. And the strip reaches a plateau $\left(\sim 60^{\circ}\right)$ of the rotation angle (Fig. 4(b) and (c)). In our simulations, we can set the gravity equal to 0 (e.g., the microgravity environment) and observe almost immediate rigid body rotation (Fig. 4(b)). The theoretical rotation limit $\left(90^{\circ}\right)$ is reached for magnetic magnitude as small as $5 \mathrm{mT}$ (Fig. 4(c)). These examples clearly show that gravity plays an important role in determining the magnetic stripe's deformation modes, which can alter the performance of the magnetic robotic jumping, turning, and swimming. Furthermore, this also calls for more systematic simulations of the nonlinear deformation of ferromagnetic structures to design more efficient magnetic robots under different working conditions, to which the newly proposed magttice model can play an important role.

\subsection{Turning of the magnetic robot}

Uniform external magnetic fields with a changing direction are usually necessary to maneuver robots for various forms of locomotion, such as rolling, jumping, and swimming. To model the homogeneous external magnetic field with a changing direction, we can link the new ( $\left.\mathbf{B}^{\text {/applied }}\right)$ and original $\left(\mathbf{B}^{\text {applied }}\right)$ applied magnetic flux densities through a rigid body rotation matrix

$$
\mathbf{B}_{i}^{\text {applied }}=R_{i j} \mathbf{B}_{j}^{\text {applied }}
$$

where $R_{i j}$ is the component of the matrix $\boldsymbol{R}$ for a given rotational angle $\Delta \theta(\Delta \theta>0$ for counter-clockwise rotation), such that

$$
\mathbf{R}=\left(\begin{array}{cc}
\cos (\Delta \theta) & -\sin (\Delta \theta) \\
\sin (\Delta \theta) & \cos (\Delta \theta)
\end{array}\right)
$$

Substituting eqn (7) and (8) into the magnetic force defined by eqn (8), the new forces associated with the rotating external magnetic field can be expressed as

$$
\left(f_{m}{ }^{\prime}\right)_{i}{ }^{a}=R_{i j}\left(f_{m}\right)_{i}{ }^{a}, \quad i, j=1,2,3, \quad a=1,2, \ldots, 8 .
$$

(a)

(b)
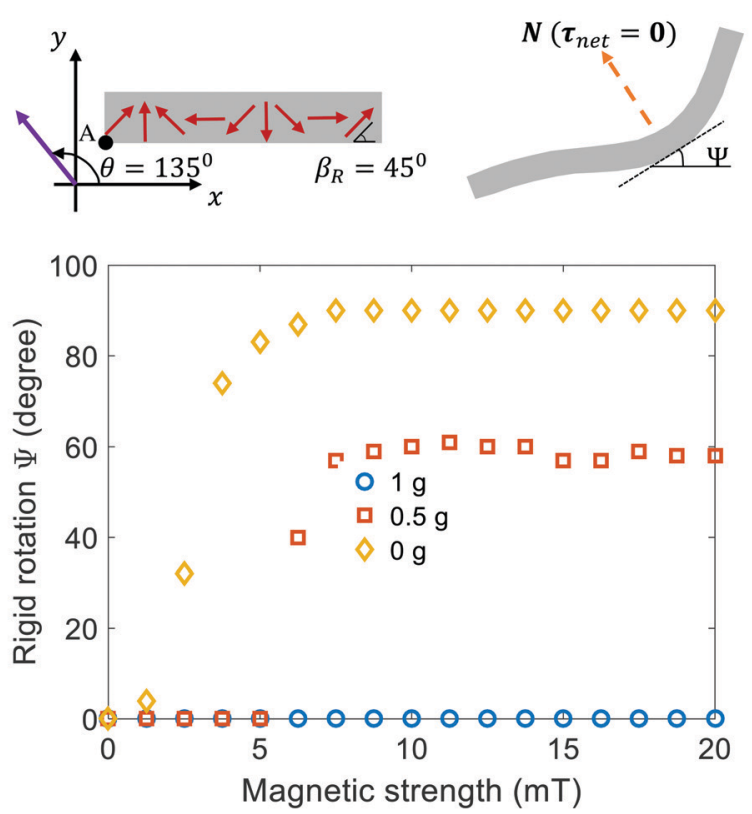

(c)

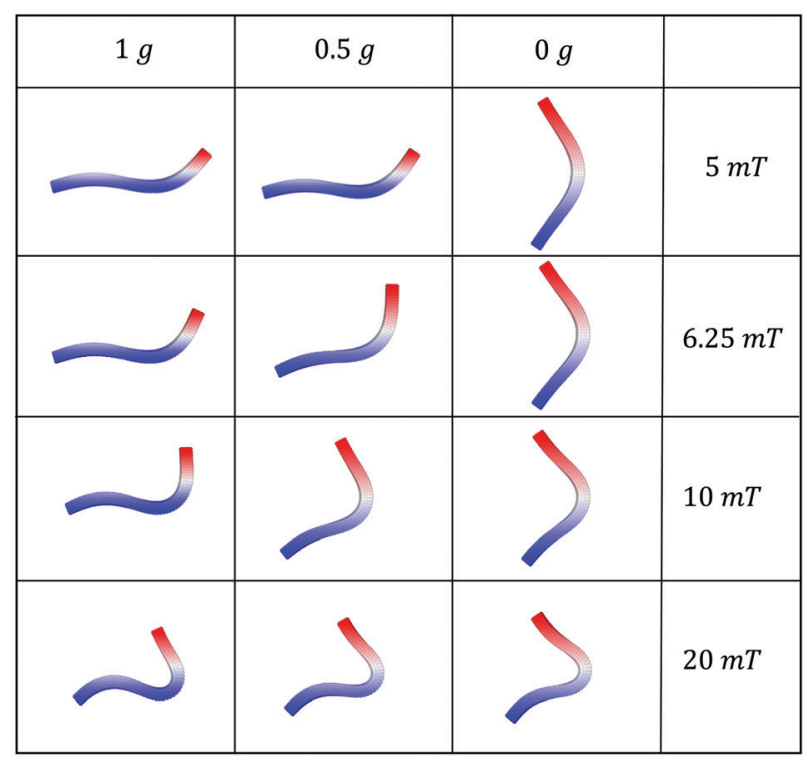

Fig. 4 Competing effect of magnetic forces and the gravity on the rigid body motion. (a) Schematics of the rigid body rotation. (b) Rigid rotation as a function of magnetic strength and gravity. (c) Representative deformed configurations of the robot under different magnetic and gravitational forces. 
We only need to compute the magnetic forces at each node once for a given $\theta$ and directly compute the new magnetic forces after rotating the external magnetic field to $\theta+\Delta \theta$ based on eqn (9). It enables us to simulate the external magnetic field's rotation by just modifying the variables in the input script of LAMMPS.

We demonstrate this function by using the robot turningdeformation as an example. The robot is first deformed into a "C" shape by setting $\theta=225^{\circ}$ and $\left|\mathbf{B}^{\text {applied }}\right|=16.9 \mathrm{mT}$. Then we perform three sequential rotations along the clockwise direction with $\Delta \theta=-45^{\circ}$. The robot is rotating with the external magnetic field and maintains the "C" shape (Fig. 5) (see Movie 1 of ESI $\dagger$ ). Following this deformation sequence, it is very interesting to note that a "C" shape can be achieved for $\theta=$ $90^{\circ}$. This is different from the " $\mathrm{C}$ " shape starting from the fixed direction of the external magnetic field (Fig. 3), indicating that the structure is bi-stable.

\subsection{Swimming of magnetic robots}

To demonstrate this magttice platform's capability to model the multiphysics problem, fluid-structure interactions, we investigate the swimming of the magnetic robot and compare it with the experimental study in terms of the swimming gaits of the soft robot. As shown in the experiments by Hu et al. ${ }^{9}$ and our simulations, the soft magnetic robot's asymmetric shape can alternate between the " $\mathrm{C}$ "- and " $\mathrm{V}$ "-shapes under periodic $B$ with time-varying magnitude along the principal axis. When the soft magnetic robot is immersed in water, this shapechanging mechanism can push it to swim upwards and overcome gravity, which manifests a gait similar to jellyfish swimming. ${ }^{15,61,62}$ In the simulation, we model the same robot used in the experimental study, with length $L=3.7 \mathrm{~mm}$ ( $x$-direction), width $w=1.5 \mathrm{~mm}$ ( $y$-direction), and thickness $h=185 \mu \mathrm{m}$ (z-direction). It is placed in a fluid domain with size $L_{x}=16 \mathrm{~mm}, L_{y}=8 \mathrm{~mm}$ and $L_{z}=16 \mathrm{~mm}$ with center initially at $\left(L_{x} / 2, L_{y} / 2, L_{z} / 4\right)$. To model the swimming motion of the robot in water, a fluid-structure interaction framework is introduced. Here, we briefly review the computational method used in this framework, which is detailed in our recent work..$^{50}$ The fluid is governed by the Navier-Stokes equation (NSE)

$$
\begin{gathered}
\frac{\partial \mathbf{u}}{\partial t}+\mathbf{u} \cdot \nabla \mathbf{u}=-\frac{1}{\rho^{f}} \nabla p+\frac{\mu}{\rho^{f}} \nabla^{2} \mathbf{u}+\mathbf{F}_{\mathrm{b}}, \\
\nabla \cdot \boldsymbol{u}=0,
\end{gathered}
$$

where $\rho^{f}, \mathbf{u}$, and $p$ are the fluid density, velocity, and pressure, respectively. $\mu$ is the dynamic viscosity of the fluid, and $\mathbf{F}_{\mathrm{b}}$ is the body force. Instead of solving the NSE directly, we use the LBM to solve the discrete Boltzmann equation that can recover incompressible NSE through Chapman-Enskog analysis, ${ }^{63}$ due to its high efficiency and accuracy. In our simulation scheme, the D3Q19 model is used ${ }^{64}$ (Fig. 6(a)), where the fluid particles have possible discrete velocities in 19 directions. The explicit parameter underpinning the LBM is density distribution function $f_{i}(\mathbf{x}, t)$, which is associated with the macroscopic properties of fluid as

$$
\rho^{f}=\sum_{i} f_{i}, \rho^{f} \mathbf{u}=\sum_{i} f_{i} \mathbf{e}_{i}+\frac{1}{2} \mathbf{F}_{\mathrm{b}} \Delta t .
$$

The magnetic robot is represented by a flat sheet and accounted for by the magttice model introduced in Section 2 . The mechanical properties of the magnetic robot are the same as those in the experimental study $(G=33 \mathrm{kPa}, K=20 \mathrm{G}$, density $\rho=1.86 \mathrm{~g} \mathrm{~cm}^{-3}$ and $\left.\frac{1}{\mu_{0}}\left|\widetilde{\mathbf{B}}^{r}\right|=62 \mathrm{kA} \mathrm{m}^{-1}\right)$. The coupling between the magnetic robot and fluid is fulfilled by the immersed boundary method (IBM), which has been extensively used to study the fluid-structure interaction problems. ${ }^{48,50,65,66,67}$ In IBM, the FSI force $\mathbf{F}^{f}$ is calculated as

$$
\mathbf{F}^{f}=\beta\left(\mathbf{u}^{f}(t)-\mathbf{u}^{s}(t)\right),
$$

where $\beta$ is the force coefficient, $\mathbf{u}^{s}(t)$ is the velocity of the structure, and $\mathbf{u}^{f}(t)$ is the fluid velocity at the position where the structure locates. $\mathbf{u}^{f}(t)$ is interpolated from its surrounding fluid velocity as

$$
\mathbf{u}^{f}(t)=\int_{\Omega} \mathbf{u}(\mathbf{x}, t) \delta\left(\mathbf{x}-\mathbf{x}^{s}\right) \mathrm{d} \Omega .
$$

where $\delta$ is a smoothed approximation of the Dirac delta interpolation function. To reflect the existence of the structure in the (a)

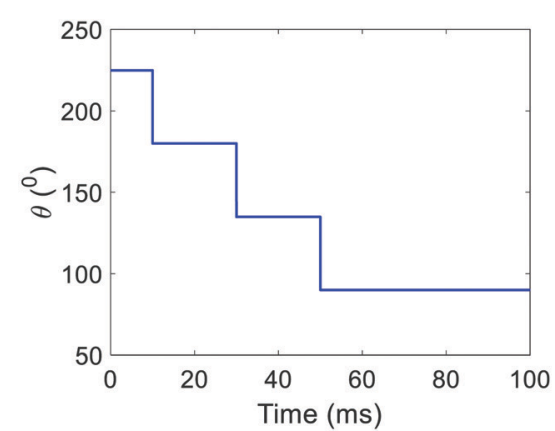

(b)

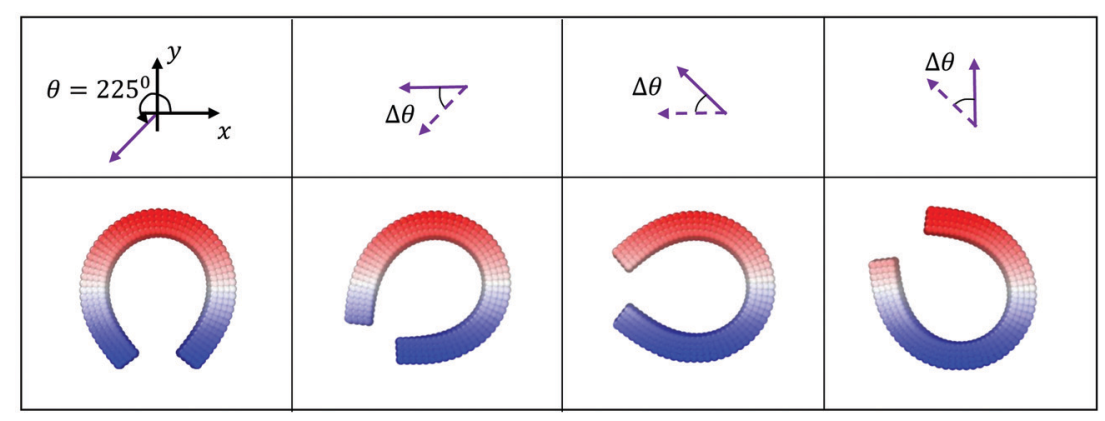

Fig. 5 (a) Time variation of the direction of the external magnetic field. (b) Structures with spatially varied residual magnetic flux for robotic-turn. Here we set $\Delta \theta=-45^{\circ}$. 
(a)
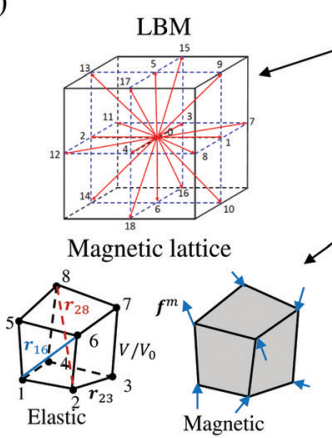

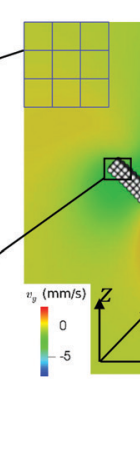

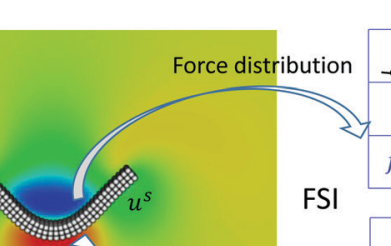

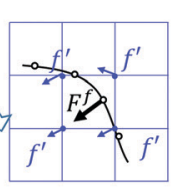

FS

(c)
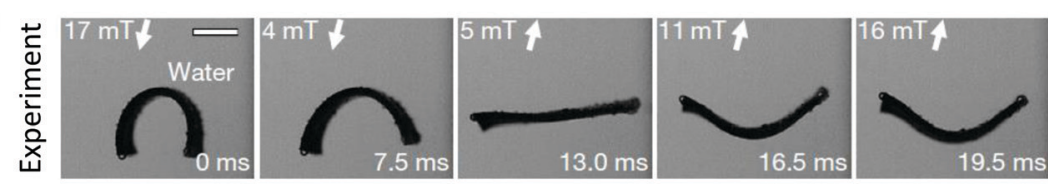
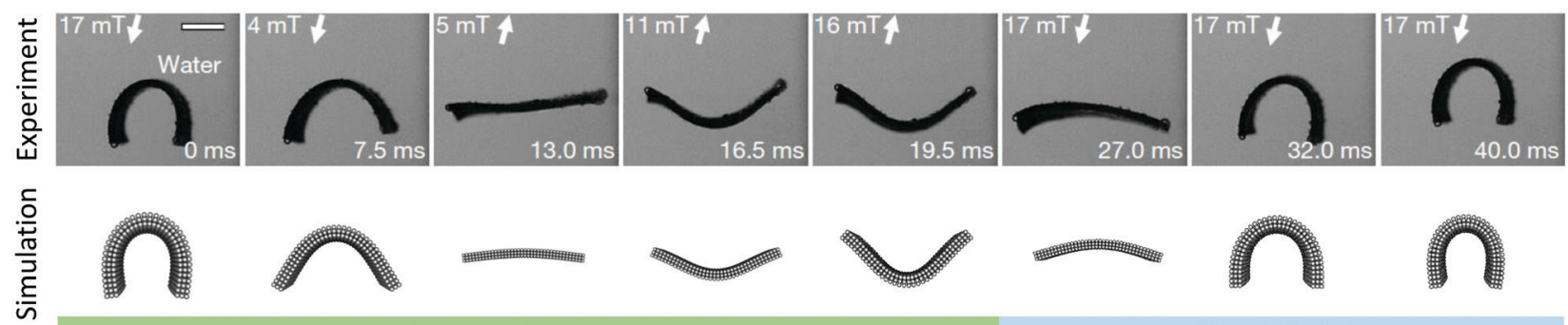

Power stroke

Fig. 6 Swimming of the magnetic robot. (a) Fluid-structure interaction scheme for the swimming of the magnetic robot. (b) Sequence of the applied magnetic field $\mathbf{B}^{\text {applied }}, \alpha$ is equal to $360^{\circ}-\theta$. (c) Comparison of the gaits of the magnetic robot in one period: from recovery stroke to power stroke. The experimental results are adapted from $\mathrm{Hu}$ et al. ${ }^{9}$

fluid, the FSI force will be spread into the surrounding fluid mesh as

$$
\mathbf{f}^{\prime}(\mathbf{x}, t)=\int_{\Omega^{s}} \mathbf{F}^{f} \delta\left(\mathbf{x}-\mathbf{x}^{s}\right) \mathrm{d} \Omega .
$$

The sequence of $\mathbf{B}^{\text {applied }}$ is given in Fig. $6(\mathrm{~b})$, where the maximum $\mathbf{B}^{\text {applied }}, \mathbf{B}_{\max }^{\text {applied }}=17 \mathrm{mT}$ and frequency $f=25 \mathrm{~Hz}$. Accordingly, the robot can perform locomotion when $B$ is oscillating along the direction $\alpha=105^{\circ}$ and $\alpha=285^{\circ}$. In one period, the prescribed $\mathbf{B}^{\text {applied }}$ sequence can make the robot demonstrate a slow recovery stroke, in which the robot changes from the "C"-shape to "V"-shape (0-19.5 ms as shown in Fig. 6(c)). Following is a fast power stroke (19.5-32 ms as shown in Fig. 6(c)), which takes the robot back to the "C"-shape. From the comparison of the configuration evolution of the magnetic robot between experiment and simulation results (cf. Fig. 6(c)), we can see that the current fluid-structure interaction platform can capture the whole swimming gaits of the robot underwater. Furthermore, the asymmetry of the " $\mathrm{C}$ "-shape and " $\mathrm{V}$ "-shape makes the robot experiences more propulsion in power stroke than the reduction in recovery stroke. This net propulsion will propel the robot to move upwards along the $z$-direction. It resembles that of an actual jellyfish swimming, ${ }^{61}$ and it is also observed in our simulation (see Movie 2 of ESI $\dagger$ ).

\section{Conclusion}

We derived a lattice model for hard-magnetic soft materials, so-called magttice. In the magttice model, the elastic energies are described by lattice stretching and volumetric changes, and the magnetic energy is modeled as potential energy associated with given nodal forces. Within this magttice model, the magnetic actuation can be incorporated into the existing lattice model in the OpenFSI package (https://github.com/huilinye/ OpenFSI) directly through variables in the job script of LAMMPS simulations. Tuning the magnitude and direction of the applied magnetic flux density can also be realized in the script, without modifying any source code, if the external magnetic field is spatially uniform. The proposed magttice model is powerful and easy to use, as demonstrated by the examples in this study, including the origami plate and magnetic robots. Besides excellent agreements with the previous FEM simulations and experiments, our numerical analysis also revealed that magnetic forces would only exist at the domain boundaries under a uniform external magnetic field if the residual magnetic flux density is uniform within the domain. This finding will help develop a more efficient reduced-order model for structures with many domain structures, such as origami plates. Besides, we found that gravity plays an important role in the soft magnetic robots' rigid body rotation. This may provide theoretical guidance for designing soft magnetic robots working in environments with different gravities, such as microgravity in outer space. Lastly, the magnetic robot swimming modeling confirms the capability of handling nonlinear and multiphysics coupled deformations, with which another path to design soft robots for biomedical applications, such as medical micro/nanorobots, is explored. ${ }^{14}$

\section{Conflicts of interest}

There are no conflicts to declare. 


\section{Acknowledgements}

H. Y. and Y. L. would like to acknowledge the support by the National Science Foundation under grant no. OAC-1755779. T. Z. acknowledges the support of the National Science Foundation under grant no. CMMI-1847149. H. Y. and Y. L. are grateful for the support from the Department of Mechanical Engineering at the University of Connecticut. Y. L. is partially supported by ASME Robert M. and Mary Haythornthwaite Research Initiation Award. T. Z. acknowledges the support from the Department of Mechanical and Aerospace Engineering at Syracuse University. H. Y. was partially supported by a fellowship grant from GE's Industrial Solutions Business Unit under a GE-UConn partnership agreement. The views and conclusions contained in this document are those of the authors and should not be interpreted as necessarily representing the official policies, either expressed or implied, of Industrial Solutions or UConn. This research also benefited in part from the computational resources and staff contributions provided by the Booth Engineering Center for Advanced Technology (BECAT) at the University of Connecticut. The authors also acknowledge the Texas Advanced Computing Center (TACC) at The University of Texas at Austin (Frontera project and the National Science Foundation award 1818253) and Comet cluster at San Diego Supercomputer Center (National Science Foundation award 1341698) (SDSC) for providing HPC resources that have contributed to the research results reported within this paper.

\section{Notes and references}

1 J. Ginder, S. Clark, W. Schlotter and M. Nichols, Int. J. Mod. Phys. B, 2002, 16, 2412-2418.

2 Y. Li, J. Li, W. Li and H. Du, Smart Mater. Struct., 2014, 23, 123001.

3 W. Li, K. Kostidis, X. Zhang and Y. Zhou, IEEE/ASME International Conference on Advanced Intelligent Mechatronics, 2009, pp. 233-238.

4 H. Böse, R. Rabindranath and J. Ehrlich, J. Intell. Mater. Syst. Struct., 2012, 23, 989-994.

5 S. Hong, Y. Jung, R. Yen, H. F. Chan, K. W. Leong, G. A. Truskey and X. Zhao, Lab. Chip, 2014, 14, 514-521.

6 S. Kashima, F. Miyasaka and K. Hirata, IEEE Trans. Magn., 2012, 48, 1649-1652.

7 S. Opie and W. Yim, J. Intell. Mater. Syst. Struct., 2011, 22, 113-125.

8 S. Wu, Q. Ze, R. Zhang, N. Hu, Y. Cheng, F. Yang and R. Zhao, ACS Appl. Mater. Interfaces, 2019, 11, 41649-41658.

9 W. Hu, G. Z. Lum, M. Mastrangeli and M. Sitti, Nature, 2018, 554, 81-85.

10 Y. Kim, G. A. Parada, S. Liu and X. Zhao, Sci. Robot, 2019, 4, 7329.

11 T. Xu, J. Zhang, M. Salehizadeh, O. Onaizah and E. Diller, Sci. Robot., 2019, 4, 4494.

12 S. Wu, C. M. Hamel, Q. Ze, F. Yang, H. J. Qi and R. Zhao, Adv. Intell. Syst., 2020, 2000060.
13 K. E. Peyer, L. Zhang and B. J. Nelson, Nanoscale, 2013, 5, 1259-1272.

14 Z. Wu, Y. Chen, D. Mukasa, O. S. Pak and W. Gao, Chem. Soc. Rev., 2020, 49, 8088-8112.

15 Z. Ren, T. Wang, W. Hu and M. Sitti, Robotics: Science and Systems, 2019.

16 G. Mao, M. Drack, M. Karami-Mosammam, D. Wirthl, T. Stockinger, R. Schwödiauer and M. Kaltenbrunner, Sci. Adv., 2020, 6, eabc0251.

17 H.-W. Huang, M. S. Sakar, A. J. Petruska, S. Pané and B. J. Nelson, Nat. Commun., 2016, 7, 1-10.

18 H. Song, H. Lee, J. Lee, J. K. Choe, S. Lee, J. Y. Yi, S. Park, J.-W. Yoo, M. S. Kwon and J. Kim, Nano Lett., 2020, 20(7), 5185-5192.

19 J. Cui, T.-Y. Huang, Z. Luo, P. Testa, H. Gu, X.-Z. Chen, B. J. Nelson and L. J. Heyderman, Nature, 2019, 575, 164-168.

20 Y. Kim, H. Yuk, R. Zhao, S. A. Chester and X. Zhao, Nature, 2018, 558, 274-279.

21 F. Fahrni, M. W. Prins and L. J. van IJzendoorn, Lab Chip, 2009, 9, 3413-3421.

22 S. Zhang, Z. Cui, Y. Wang and J. den Toonder, Lab Chip, 2020, 20, 3569-3581.

23 S. Khaderi, C. Craus, J. Hussong, N. Schorr, J. Belardi, J. Westerweel, O. Prucker, J. Rühe, J. D. Toonder and P. Onck, Lab Chip, 2011, 11, 2002-2010.

24 J. Belardi, N. Schorr, O. Prucker and J. Rühe, Adv. Funct. Mater., 2011, 21, 3314-3320.

25 H. Gu, Q. Boehler, H. Cui, E. Secchi, G. Savorana, C. De Marco, S. Gervasoni, Q. Peyron, T.-Y. Huang and S. Pane, et al., Nat. Commun., 2020, 11, 1-10.

26 X. Zhao, J. Kim, C. A. Cezar, N. Huebsch, K. Lee, K. Bouhadir and D. J. Mooney, Proc. Natl. Acad. Sci. U. S. A., 2011, 108, 67-72.

27 H. K. Yap, J. H. Lim, F. Nasrallah, F.-Z. Low, J. C. Goh and R. C. Yeow, IEEE International Conference on Rehabilitation Robotics (ICORR), 2015, pp. 735-740.

28 P. R. Buckley, G. H. McKinley, T. S. Wilson, W. Small, W. J. Benett, J. P. Bearinger, M. W. McElfresh and D. J. Maitland, IEEE Trans. Biomed. Eng., 2006, 53, 2075-2083.

29 H. Gu, S. W. Lee, J. Carnicelli, T. Zhang and D. Ren, Nat. Commun., 2020, 11, 1-11.

30 S. Zhang, P. Zuo, Y. Wang, P. R. Onck and J. M. den Toonder, ACS Appl. Mater. Interfaces, 2020, 12(24), 27726-27736.

31 K. Yu, N. X. Fang, G. Huang and Q. Wang, Adv. Mater., 2018, 30, 1706348.

32 R. Zhao, Y. Kim, S. A. Chester, P. Sharma and X. Zhao, J. Mech. Phys. Solids, 2019, 124, 244-263.

33 G. Z. Lum, Z. Ye, X. Dong, H. Marvi, O. Erin, W. Hu and M. Sitti, Proc. Natl. Acad. Sci. U. S. A., 2016, 113, E6007-E6015.

34 J. Li, T. D. Pallicity, V. Slesarenko, A. Goshkoderia and S. Rudykh, Adv. Mater., 2019, 31, 1807309.

35 A. Goshkoderia, V. Chen, J. Li, A. Juhl, P. Buskohl and S. Rudykh, Phys. Rev. Lett., 2020, 124, 158002. 
36 S. M. Montgomery, S. Wu, X. Kuang, C. D. Armstrong, C. Zemelka, Q. Ze, R. Zhang, R. Zhao and H. J. Qi, 2020, arXiv preprint arXiv:2006.12721.

37 K. H. Lee, K. Yu, H. Al Ba'ba'a, A. Xin, Z. Feng and Q. Wang, Research, 2020, 2020, 4825185.

38 W. Gao, D. Kagan, O. S. Pak, C. Clawson, S. Campuzano, E. Chuluun-Erdene, E. Shipton, E. E. Fullerton, L. Zhang and E. Lauga, et al., Small, 2012, 8, 460-467.

39 K. Danas, S. Kankanala and N. Triantafyllidis, J. Mech. Phys. Solids, 2012, 60, 120-138.

40 M. Ostoja-Starzewski, Appl. Mech. Rev., 2002, 55, 35-60.

41 X. He, M. Aizenberg, O. Kuksenok, L. D. Zarzar, A. Shastri, A. C. Balazs and J. Aizenberg, Nature, 2012, 487, 214-218.

42 O. Kuksenok and A. C. Balazs, Sci. Rep., 2015, 5, 1-7.

43 O. Kuksenok and A. C. Balazs, Mater. Horiz., 2016, 3, 53-62.

44 A. Alexeev, R. Verberg and A. C. Balazs, Macromolecules, 2005, 38, 10244-10260.

45 V. V. Yashin and A. C. Balazs, J. Chem. Phys., 2007, 126, 124707.

46 T. Zhang, Extreme Mech. Lett., 2019, 26, 40-45.

47 P. Dayal, O. Kuksenok and A. C. Balazs, Langmuir, 2009, 25, 4298-4301.

48 H. Ye, Z. Shen and Y. Li, Comput. Mech., 2018, 62, 457-476.

49 G. A. Buxton, R. Verberg, D. Jasnow and A. C. Balazs, Phys. Rev. E: Stat., Nonlinear, Soft Matter Phys., 2005, 71, 056707.

50 H. Ye, Z. Shen, W. Xian, T. Zhang, S. Tang and Y. Li, Comput. Phys. Commun., 2020, 256, 107463.

51 A. Dorfmann and R. Ogden, Eur. J. Mech. A Solids, 2003, 22, 497-507.

52 S. Kankanala and N. Triantafyllidis, J. Mech. Phys. Solids, 2004, 52, 2869-2908.
53 T. K. Kim, J. K. Kim and O. C. Jeong, Microelectron. Eng., 2011, 88, 1982-1985.

54 P. Saxena, M. Hossain and P. Steinmann, Int. J. Solids Struct., 2013, 50, 3886-3897.

55 D. Garcia-Gonzalez, Smart Mater. Struct., 2019, 28, 085020.

56 T. Belytschko, W. K. Liu, B. Moran and K. Elkhodary, Nonlinear finite elements for continua and structures, John wiley \& sons, 2013.

57 S. Tang, Y. Li, Y. Yang and Z. Guo, Soft Matter, 2015, 11, 7911-7919.

58 Z. Zhou, Y. Li, W. Wong, T. Guo, S. Tang and J. Luo, Soft Matter, 2017, 13, 6011-6020.

59 L. Dorfmann and R. W. Ogden, Nonlinear theory of electroelastic and magnetoelastic interactions, Springer, 2014, vol. 1.

60 L. Wang, Y. Kim, C. F. Guo and X. Zhao, J. Mech. Phys. Solids, 2020, 104045.

61 B. J. Gemmell, J. H. Costello, S. P. Colin, C. J. Stewart, J. O. Dabiri, D. Tafti and S. Priya, Proc. Natl. Acad. Sci. U. S. A., 2013, 110, 17904-17909.

62 Z. Ren, W. Hu, X. Dong and M. Sitti, Nat. Commun., 2019, 10, 1-12.

63 S. Succi, The lattice Boltzmann equation: for fluid dynamics and beyond, Oxford University Press, 2001.

64 F. Mackay, S. T. Ollila and C. Denniston, Comput. Phys. Commun., 2013, 184, 2021-2031.

65 C. S. Peskin, Acta Numer., 2002, 11, 479-517.

66 F.-B. Tian, H. Luo, L. Zhu, J. C. Liao and X.-Y. Lu, J. Comput. Phys., 2011, 230, 7266-7283.

67 H. Ye, H. Wei, H. Huang and X.-Y. Lu, Phys. Fluids, 2017, 29, 021902. 